\title{
Designing of 3-Dimensional Underwater Fish Observation System and Experimental Research
}

\author{
Minhui Zheng ${ }^{1,2}$, Junyi Yang ${ }^{2}$, Dinghao Feng ${ }^{3}$, Dongqin Cheng ${ }^{3}$, Xi Zhang ${ }^{3,4}$ \\ 1 Faculty of Resource, China University of Geosciences, Wuhan 430074, China \\ 2 Second Institute of Oceanography, SOA, Hangzhou 310012, China
}

3 School of Mechatronics Engineering and Automation, Shanghai University, Shanghai 200072, China

4 Shanghai Key Laboratory of Intelligent Manufacturing and Robotics, Shanghai 200072

Keywords: deep sea; 3D vision; calibration; refraction.

\begin{abstract}
Underwater imaging is an important method to discover and study deep sea life. However, the information obtained from the 2-dimensional imaging materials acquired by the current technological method is too limited to meet the requirements of scientific research. In this article, a package of underwater lighting and stereo vision measurement hardware system is designed that could be carried by underwater platform. The feasibility and accuracy of the proposed methods is proved by the pool principle experiment. This system could provide technological support for the deep sea biology study and the exploitation of deep sea biological resources, and also be used for the $3 \mathrm{D}$ reconstruction and measurement of underwater micro topography and targets.
\end{abstract}

\section{Introduction}

Deep sea is abundant in natural resources and is also an important window to know the origin of life and the internal world of the earth. With the development of ROV, manned submersibles, cabled ocean observatory systems and other underwater platforms, human's observation and study of deep sea life develops from trawling to underwater camera. However, there are still many limits in applying underwater imaging materials in deep water biological studies because existing deep water observation system basically uses a single camera to take the pictures of deep water life, which can only get 2D images. Without the position information of underwater targets, the body length, the body depth and other important morphological characters necessary for the morphological classification and identification can't be calculated from the images. This limits the application of underwater camera significantly in deep sea biological resources and deep sea mineral resources research.

Stereo vision system is an effective method to quantitatively analyze features of underwater life from the deep sea imaging materials. By acquiring the image of the object with a pre-calibrated dual-camera system, the 3D information of the object could be calcuated from the images [1-6]. In this article, targeting at the requirements of deep sea biological resources survey which includes the 3D size measurement of fish, we established a package of underwater lighting and binocular vision measurement system that could be carried by underwater platform. The research results of this article could provide technological support for deep sea studies and the exploitation of deep sea biological resources.

\section{Underwater refraction camera model}

Imaging processing in the air medium can be described by the traditional pin-hole camera model. As for underwater imaging, the light from the water medium penetrates to the air medium through sealed glass, and in the end penetrates the lens and then is received by the CCD sensor. As refraction occurs when the light enters a medium from another medium which is a non-single viewpoint problem, if the camera model for the air medium is applied directly in the underwater 
condition, there will be measurement errors inevitably. Fig.1 shows the differences between the two kinds of camera models.

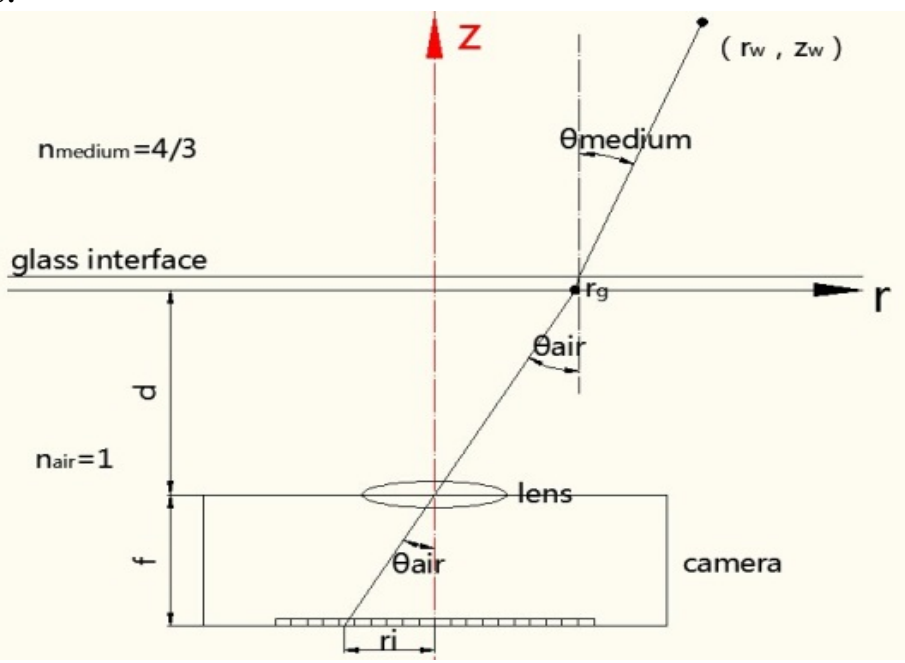

Fig.1: Underwater imaging model

According to the derivation in literatures[7], the camera model in the water medium can be established (Eq. 1). There is the complex relationship between an object point (rw, zw) under the world coordinate system and image point ri relative to $d, f$ and $n$. Here d denotes the distance between the center of lens and the surface of sealed glass. $\mathrm{f}$ is the distance between the center of the lens and CCD sensor. $\mathrm{n}$ is the index of refraction in water. As the distance between the center of the lens and the surface of the sealed glass and the refraction index of water medium are involved, the camera model in water is more complicated than in air medium (Eq. 2).

$$
\begin{aligned}
& \left(\mathrm{r}_{w}-\frac{d}{f} \mathrm{r}_{i}\right)^{2}\left[\left(\frac{f \cdot n}{r_{i}}\right)^{2}+\left(n^{2}-1\right)\right]=z_{w}{ }^{2} \\
& \frac{r_{i}}{f}=\frac{r_{w}}{Z_{w}}
\end{aligned}
$$

It can be seen that underwater camera model shown in Eq. 1 is equivalent to the pin-hole model when $d=0$ and $\theta<5^{\circ}$. Under this occasion, the camera model is equivalent to a perspective pinhole camera model with $f_{\text {effective }}=n_{\text {medium }} \cdot f$.

\section{Pool experiment and result analysis}

\subsection{Experimental system}

The artificial pool with the experimental environment of $2 \mathrm{~m} \times 4 \mathrm{~m}$ (Fig.2.a), adopted color industrial camera with two megapixels and LED flash lamps (Fig.2.b), both of which were sealed in the cylindrical shape and operated with $6000 \mathrm{~m}$ dielectric strength test. The collecting mode of the system was trigger collection to make sure that the flash lights work simultaneously with the cameras. The measured distance of the system was between $1.3 \mathrm{~m}$ to $1.7 \mathrm{~m}$. The target with checkerboard pattern is used for camera calibration. 


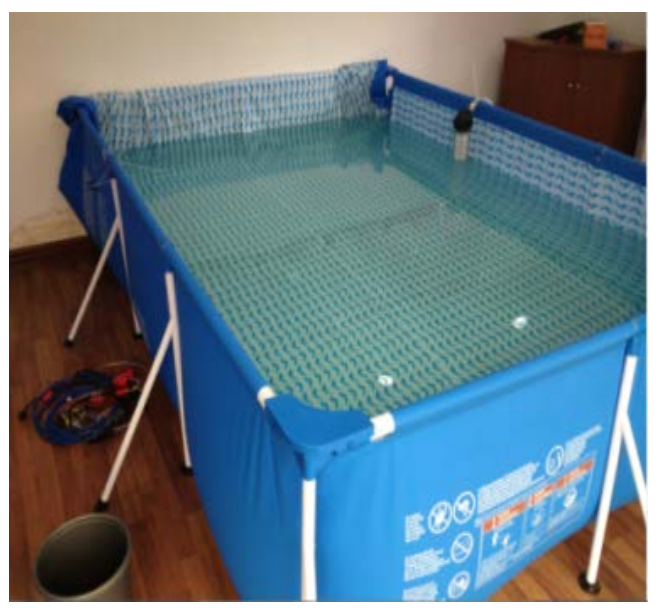

(a) Pool used in the experiment

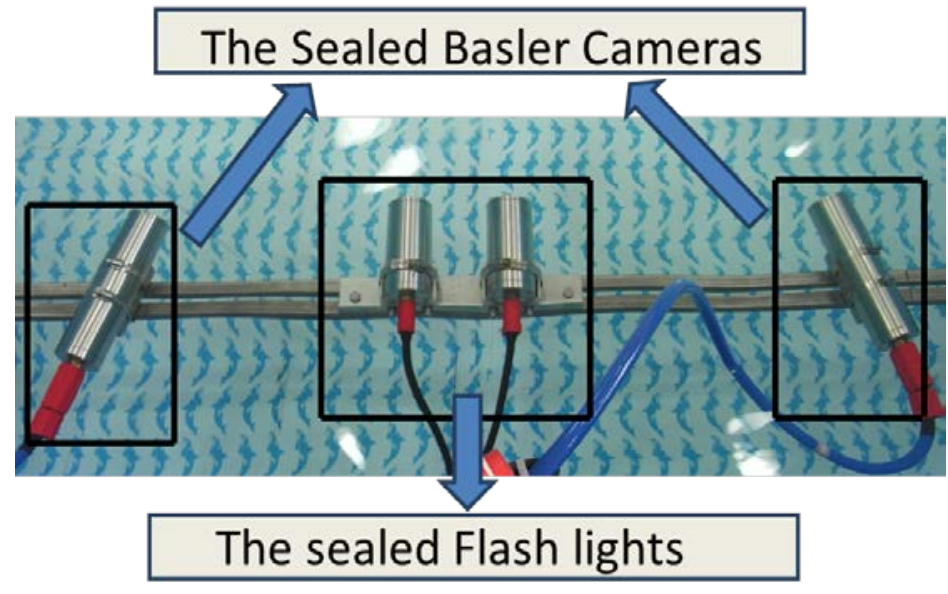

(b)Underwater binocular vision system

Fig.2: Experimental setup

Two experiments were conducted. One is the calibration experiment of the cameras under water, and the other is the dimension measurement of fish. In the calibration experiment, the calibration plate was put in different positions (Fig.3) in the view field of the camera. The physiological indexes of emulational carp were measured in the fish measurement experiment.

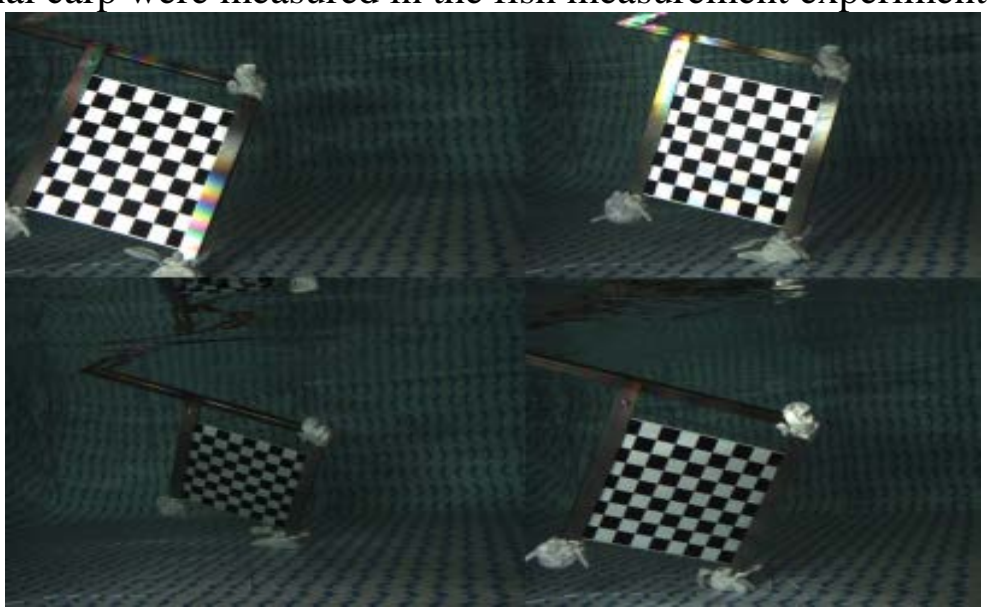

Fig.3: Underwater calibration imaging

\subsection{Calibration experiment of underwater camera and result discussion}

The cameras were calibrated in the water and in the air respectively. According to the experimental data, the obvious difference of calibration results between underwater condition and that in the air was the change of $\mathrm{fx}$ and fy. The comparison result was shown in Table 1. According to the calculated result, the focal length of the underwater camera/ the focal length of camera in the air $\approx \mathrm{n}$ water, the index of refraction is about 1.33333 .

Table 1: Comparison of calibration data

\begin{tabular}{ccc}
\hline & $\mathrm{f}_{\mathrm{x}}$ & $\mathrm{f}_{\mathrm{y}}$ \\
\hline $\begin{array}{c}\text { Underwater } \\
\text { calibration }\end{array}$ & 2501.24860079 & 2504.71654955 \\
Calibration in the air & 1875.43537389 & 1871.84691802 \\
$\mathrm{f}_{\mathrm{x}} 1 / \mathrm{f}_{\mathrm{x}} 2$ & 1.333689 & 1.338321 \\
\hline
\end{tabular}




\subsection{Verification of system measurement accuracy}

In this article, we proved the measurement accuracy of the system by measuring the size of calibrated checkerboard. The actual size of each checkerboard is $30 \mathrm{~mm}$. The sizes of checkerboards are measured on four groups of images in the horizontal and longitudinal direction respectively. According to the results, the measurement accuracy of this underwater stereo vision system was better than $0.4 \mathrm{~mm}$.

\subsection{Measurement experiment of fish and result discussion}

As for the measurement of emulational carp, the measurement range was between $1.3 \mathrm{~m}$ to $1.7 \mathrm{~m}$. The measurement target was the emulational carp shown in Fig.4.b. To measure the emulational carp, a feature point is selected manually from the left camera image. Then the accurate matching point on the right camera image is determined by providing an initial matching manually. Finally, the 3D coordinate was calculated according to the camera calibration parameters. The measurement results were shown in Table 2. According to the result, the measurement accuracy is better than $2.7 \mathrm{~mm}$
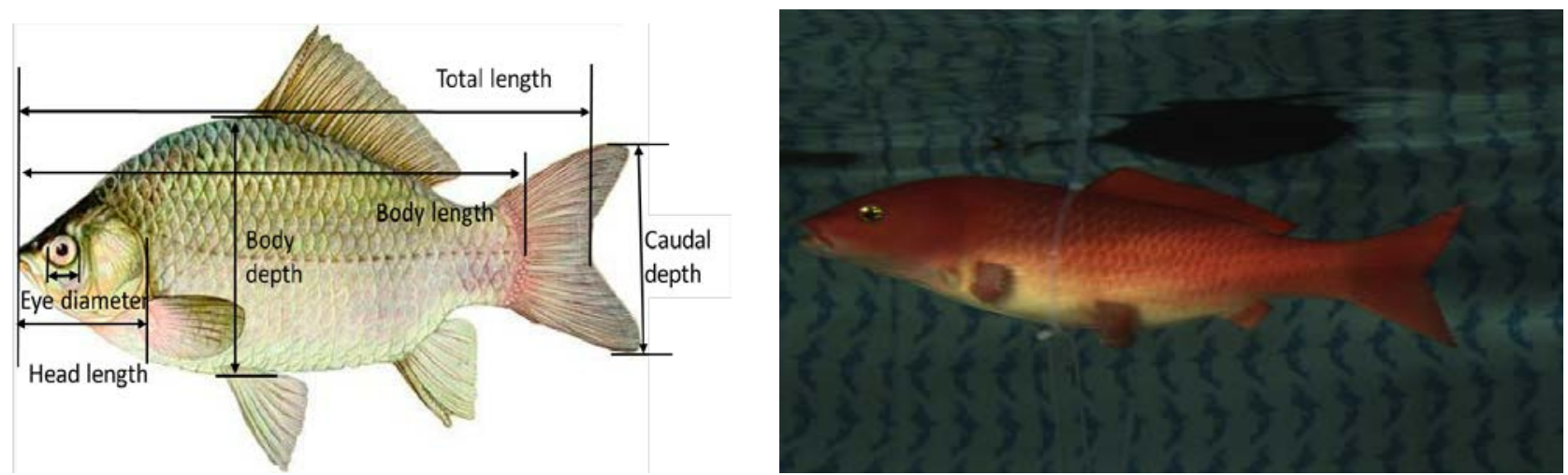

(a) Diagram of the morphological characters of fish (b) Emulational carp

Fig.4: Emulational carp for measurement

According to the underwater camera calibration experiment and the fish measurement experiment, it can be found that measurement with emulational carp caused larger errors than with the checkerboard calibration. This result was mainly caused by the selection of feature dots. The characteristic of the checkerboard was the corner point, which was easy to select. However, the feature dot of an emulational fish was not obvious and manual selection would bring some errors.

Table 2: Measurement data of emulational carp experiment

\begin{tabular}{|c|c|c|c|c|c|c|c|}
\hline \multirow[t]{2}{*}{ Parameters } & \multicolumn{4}{|c|}{ Actual measurement value[mm] } & \multirow{2}{*}{$\begin{array}{l}\text { Mean } \\
{[\mathrm{mm}]}\end{array}$} & \multirow{2}{*}{$\begin{array}{l}\text { Actual } \\
\text { size } \\
{[\mathrm{mm}]}\end{array}$} & \multirow{2}{*}{$\begin{array}{c}\text { Difference } \\
{[\mathrm{mm}]}\end{array}$} \\
\hline & 1 & 2 & 3 & 4 & & & \\
\hline Body length & 283.2 & 285.8 & 289.6 & 288.7 & 286.8 & 285.4 & 1.4 \\
\hline Fork length & 319.2 & 317.9 & 321.7 & 320.5 & 319.8 & 322.5 & -2.7 \\
\hline Body width & 99.7 & 101.1 & 101.0 & 96.9 & 99.7 & 101.6 & -1.9 \\
\hline $\begin{array}{l}\text { Tail fin } \\
\text { height }\end{array}$ & 100.9 & 101.0 & 101.3 & 101.4 & 101.1 & 103.8 & -2.7 \\
\hline Eye diameter & 10.8 & 11.2 & 11.4 & 11.9 & 11.3 & 12.3 & -1.0 \\
\hline Head length & 85.8 & 80.3 & 86.6 & 83.2 & 84.0 & 84.6 & -0.6 \\
\hline
\end{tabular}




\section{Conclusions}

The stereo vision method is proposed to measure the 3D sizes of deep sea fish in this article. We designed a package of binocular vision measurement and underwater lighting system that could be carried with deep sea platform. Through pool experiment, we completed the underwater camera calibration and measurement of the characteristic sizes of fish. The experimental results proved that this system could be used to measure the biological characteristic data of fish. In the future, we plan to perform a deep sea field measurement to provide data support for the further improvement of this system.

\section{Acknowledgements}

This work was financially supported by the National High Technology Research and Development Program of China(863 Program)(2012AA092101, 2012AA092102) and the Subject of National Natural Science Foundation of China(41376169).

\section{References}

[1] Klimley A. P. and Brown S. T., Stereophotography for the field biologist: measurement of lengths and three-dimensional positions of free-swimming sharks. Marine Biology, 74(2), pp. 175-185, 1983.

[2] Naiberg A. and Little J.J., A unified recognition and stereo vision system for size assessment of fish. Proceedings of the Second IEEE Workshop on Applications of Computer Vision, pp. 2-9, 1994.

[3] Harvey E. and Shortis M., A system for stereo-video measurement of sub-tidal organisms. Marine Technology Society Journal, Vol. 29, pp. 10-22, 1996.

[4] Harvey E., Cappo M., Shortis M. et al., The accuracy and precision of underwater measurements of length and maximum body depth of southern bluefin tuna (Thunnus maccoyii) with a stereovideo camera system. Fisheries Research, 63(3), pp. 315-326, 2003.

[5] Butail S. and Paley D.A., Three-dimensional reconstruction of the fast-start swimming kinematics of densely schooling fish. Journal of the Royal Society Interface, 9(66), pp. 77-88, 2012.

[6] Cappo M., Speare P. and De'ath G., Comparison of baited remote underwater video stations (BRUVS) and prawn (shrimp) trawls for assessments of fish biodiversity in inter-reefal areas of the Great Barrier Reef Marine Park. Journal of Experimental Marine Biology and Ecology, 302(2), pp. 123-152, 2004.

[7] Tali T., Yoav S., Clayton K. et al., "Flat Refractive Geometry," IEEE Transactions on Pattern Analysis and Machine Intelligence, 34(1), pp. 51-65, 2012. 